\title{
SCREENING AND CONFIRMATION OF DIFFERENT SYNTHETIC ADULTERANTS IN SLIMMING PRODUCTS
}

\author{
SAJAD FAKHRI ${ }^{1}$, BAHAREH MOHAMMADI ${ }^{2}$, RONAK JALILI ${ }^{2}$ MARZIYEH HAJIALYANI ${ }^{3}$, GHOLAMREZA BAHRAMI $^{1,2 *}$ \\ ${ }^{1}$ Department of Pharmacology and Toxicology, School of Pharmacy, Kermanshah University of Medical Science, Kermanshah, Iran. \\ ${ }^{2}$ Medical Biology Research Center, Kermanshah University of Medical Science, Kermanshah, Iran. ${ }^{3}$ Pharmaceutical Sciences Research \\ Center, Kermanshah University of Medical Science, Kermanshah, Iran. Email: gbahrami@kums.ac.ir
}

Received: 11 September 2017, Revised and Accepted: 15 November 2017

ABSTRACT

Objective: The presence of synthetic drugs (as adulterant) in slimming herbal products of world markets has contravened the country's law and has created many troubles for some consumers of slimming products. This study aims to use a simple and reliable method for simultaneously finding and validating the presence of possible adulterants in some slimming products, which could not be discriminated due to misleading packaging.

Method: Fifteen slimming products were obtained from the Iran pharmaceutical markets. Liquid chromatography-ultraviolet and LC-mass spectrometry methods were performed for simultaneous screening and confirmation of adulterants.

Results: The most abundant adulterant was Phenolphthalein, which has been used in 11 cases of the 15 slimming products. Furthermore, 5 products included caffeine, two included phendimetrazine, and two included protriptyline.

Conclusion: The performed method was successful in detection of different adulterants. According to the presence of synthetic drugs in slimming herbal products, and their threats to people's health it is necessary to create awareness in this case through public authorities.

Keywords: Obesity, Mass spectroscopy, Slimming products, Adulterants, Liquid chromatography.

(C) 2018 The Authors. Published by Innovare Academic Sciences Pvt Ltd. This is an open access article under the CC BY license (http://creativecommons. org/licenses/by/4. 0/) DOI: http://dx.doi.org/10.22159/ajpcr.2018.v11i2.22516

\section{INTRODUCTION}

Recently, consumption of dietary supplements containing medicinal ingredients is associated with some serious health problems due to the presence of illegal adulterants. Hence, detection of these illegal ingredients has been recognized as a worldwide crisis to confirm the purity of commercially dietary supplements. Botanical dietary supplements have attracted more attention due to the general perceptions that they are safe and harmless and cause no detrimental side effects of synthetic products and chemical agents. The prevalence of obesity, as a major risk for public health, has caused increasingly demand for anti-obesity supplements worldwide. Increasing body fat mass along the time improves the possibility of being affected by osteoarthritis, joint damage, gallstone [1], thrombosis [2], cancers [3] and many other endocrines and metabolic diseases, cardiovascular diseases [4,5], nervous system, and neurological diseases [6,7]. Since these problems vigorously affect the public health, it could be necessary to use safe and harmless weight-loss supplements. Natural anti-obesity products have been always considered as attractive alternatives to synthetic products as they are publically advertised as products containing purely natural ingredients with lacking specific side effects $[8,9]$. The truth is that such herbal products seem to be weaker than synthetic slimming products and it could be nearly impossible to achieve rapid short-term effects, therefore some manufacturers often illegally add synthetic pharmaceutical drugs to the natural product to improve their efficacy and achieve huge profits, while the presence of these ingredients could not be discriminated due to misleading packaging [10-13]. Unfortunately, continuous consumption of chemical slimming products, which are illegally adulterated with synthetic materials, may cause severe harms to patients and could be considered as a threat to individual's health and even a reason for mortality [14].

Using such products, patients may face several intensive physical problems such as insanity, cardiovascular, and liver diseases [15-17].
Studies reported several cases of renal failure have due to consumption of these adulterated slimming products [18-20].

The most common adulterant found in herbal products includes antiappetite derived from amphetamines, which cause a reduction in intestinal fat absorption [9]. Studies showed that about $80 \%$ of slimming products illegally available in the market include amphetamine derivatives [21]. Beyond amphetamines, benzodiazepines, some coadjuvants including diazepam, flurazepam, midazolam, and chlordiazepoxide antidepression compounds (fluoxetine), diuretics (furosemide, spironolactone, and hydrochlorothiazide), and laxatives (phenolphthalein) are only a few number of undeclared pharmaceuticals identified in slimming products [9].

Considering the small number of studies on investigating adulterant in slimming products in Iran, in the present study, a simple and reliable method was performed to identify experimentally the possible adulteration cases in slimming supplements which have been commercialized in Iran, by simultaneous using highly sensitive instruments such as liquid chromatography-ultraviolet (LC-UV [22]) and liquid chromatography-mass (LC-mass) [23].

This simple method allowed the screening and confirmation of adulterants present in the slimming supplements simultaneously, based on the specific retention time of each ingredient and some extra information in their spectra.

\section{METHODS}

Samples

Fifteen commonly used slimming products (the most commonly used ones) in Iran, which were advertized in the Persian language on satellite channels and internet, were collected from herbalists and markets. Such media advertisements, which were mainly from China 
and some Southeast Asia countries, persuade people to make use of the products, mainly known to be herbal with no label indication on the presence of synthetic drugs. These products dosage forms were hard capsules (Magic Slim, Relacore, Natural Slimming, Fast Slimming, PX-1, Slimming, Natural Max Slimming, Super Slim, Weight Killer, Feel Slim, HydroxyStim) and soft capsules (Lipo-6, Botanical Slimming, Sample 12, Hodia-P57). The detection of slimming products was established by coupled LC-MS methods.

\section{Chemicals}

Standards of sibutramine, methamphetamine, orlistat, ephedrine, phenylpropanolamine, caffeine, tetrahydrocannabinol, sildenafil, phenolphthalein, phenytoin, phenobarbital, fluoxetine, fluvoxamine, some benzodiazepines, levothyroxine, and propylthiouracil (PTU) were obtained by Sigma-Aldrich. The acetonitrile and formic acid were obtained from Merck. All the used reagents were of analytical grade.

\section{Sample preparation}

We randomly selected one capsule (hard or soft) from each slimming product. A small amount of each homogenized capsule, extracted with $2 \mathrm{~mL}$ acetonitrile for $30 \mathrm{~min}$ in round bottom test tube using laboratory rotator. The extract was centrifuged ( $5 \mathrm{~min}$ at $4000 \mathrm{rpm}$ ). The supernatant was collected for examination. Then, about $5 \mu \mathrm{L}$ of the top layer of the compound was injected to LC device. Then, highperformance liquid chromatography (HPLC) and mass conditions were defined to achieve the highest level of centrifugation and determine retention time, simultaneously. Along with this, the present study made use of the mass device, in addition to HPLC and UV detector to strengthen the obtained results $[15,16,18,19]$.

\section{Measurement}

The separation process of the analytes was carried out isocratically on a MZ perfect Sill target C18 column (125.0 $\mathrm{mm} \times 4.0 \mathrm{~mm}$ ID., $5 \mu \mathrm{m}$ ) using a C18 guard column (MZ-Analysentechnik GmbH, Germany). The HPLC conditions were optimized to achieve the maximum response and the best peak shape and resolution. Different eluting programs were applied for mobile phase, using acetonitrile, water, and formic acid. The column temperature was maintained at $40^{\circ} \mathrm{C}$, and a mixture of acetonitrile, water, and formic acid (41:59:0.06 w/w/w) was eluted as a mobile phase at a flow rate of $0.7 \mathrm{~mL} / \mathrm{min}$. Qualitative analyses were performed using an Agilent 1200 series LC system consisting of a quaternary delivery pump and a thermostated column compartment. The mass analyses were performed with an Agilent 6410 triple quadrupole mass spectrometer (Agilent Technologies, Palo Alto, CA, USA) which was run by Agilent Mass Hunter Workstation B.01.03. Ionization (ESI) bin the negative mode with the capillary voltage 4000 V. Nitrogen was used as nebulizer at the pressure of $40 \mathrm{psi}$ and a source temperature of $100^{\circ} \mathrm{C}$. Drying gas (nitrogen) was heated to $300^{\circ} \mathrm{C}$ and delivered at a flow rate of $10 \mathrm{~L} / \mathrm{min}$.

\section{RESULTS AND DISCUSSION}

\section{The surface features of the samples}

Samples provided as slimming products were all in capsule shapes. After breaking the capsules, the surface features of the products were studied, considering the color, size, and shape of the powder, homogeneity of powder, etc.

Samples 11 and 14 included oil similar to soft capsules. Sample number 9 contained hard capsules filled with oil, and sample 12 contained soft capsules filled with dark color solid (Table 1). None of the samples produced specific smelling, though.

\section{Results of chromatographic analyses}

In this study, the presence of synthetic drugs in 15 commercialized slimming products of Iran market was investigated, which were in spite of the manufacturer's claim that their products contained only the extracts of the plants mentioned on the label. Identification was performed based on four criteria: Retention time, UV spectrum, and molecular ions observed in mass spectrum analysis, and the mechanism of action of each standard in losing weight. The retention times of the selected standard adulterants were compared with those of investigated slimming products. To further ensuring the reliability of results, and after assuring the similarity in retention times of the standard materials to the investigated slimming products, the UV spectra of both materials were compared. Finally, mass $\mathrm{m} / \mathrm{z}$ of investigated slimming supplements was compared to standard mass spectrum.

Analysis of sample number 1 "Majic Slim" revealed the presence of caffeine and phenolphthalein as synthetic materials. Two peaks were observed at times 2.65 and $8.93 \mathrm{~min}$, corresponding to the retention time of standard caffeine and phenolphthalein, respectively.

This could identify the presence of caffeine and phenolphthalein in product 1 and could be confirmed by mass spectroscopy analysis. The mass spectrum of sample 1 in UV range exhibited two peaks, one the molecular ion peak of phenolphthalein at m/z 318 and another the base peak at $\mathrm{m} / \mathrm{z} 274$, corresponding to the fragmentation of phenolphthalein and elimination of $\mathrm{CO}_{2}$ from the lactone ring and formation of resonance stabilized ion (Fig. 1). These characteristic peaks in association with the study of retention times, confirmed the presence of phenolphthalein and Caffeine in the structure of product number 1 . Phenolphthalein was also found as an adulterant presented in 10 other products and was identified in the products number 2,3 , $5,6,7,8,9,11,12,13$, and 14). Caffeine was also included in products number $5,9,10,12,14$, and 15 (Table 1 ).

The retention times and mass spectrum of "Relacore" as sample number 2 of slimming products showed the existence of sibutramine. The observed peak at retention time of 4.5 was attributed to the presence of sibutramine. Sibutramine is a FDA approved anti-obesity compound which has been withdrawn from the market after approving its clinical side effects on cardiovascular functions such as myocardial infarction, cardiac arrhythmia, uncontrolled hypertension, and stroke [24,25]. In addition to product number 3 , sibutramine was identified in the six other products $[4,6-8,13,14]$.

Comparison between the retention time of protriptyline and the retention time peaks observed for the slimming products, it was found that three slimming products $[4,6,8]$ were adulterated with protriptyline. The retention time of protriptyline is $10.75 \mathrm{~min}$. To confirm the presence of protriptyline as adulterant, the mass spectra of these products were examined. As an example, the mass diagram of product 4 is demonstrated in Fig. 2. The presence of lines at $\mathrm{m} / \mathrm{z} 263.4$, attributing to the molecular ion of protriptyline, and at $\mathrm{m} / \mathrm{z} 204.6$ as the fragment of protriptyline due to loss of propylamine, in the mass diagram of product number 4 confirmed that this product illegally includes protriptyline as an adulterant. Sildenafil was another adulterant presented in product 4 . The retention time at $2.19 \mathrm{~min}$, identified the presence of sildenafil in the product 4 . Considering that the molecular weight of standard sildenafil is $474.6 \mathrm{Da}$, the presence of the peak at m/z 474 in the mass spectrum of the product identified the molecular ion of sildenafil (Fig. 2). Furthermore, the existence of three peaks at $\mathrm{m} / \mathrm{z} 379.2, \mathrm{~m} / \mathrm{z} 310.6$, and $\mathrm{m} / \mathrm{z} 283$ in the mass of standard sildenafil and the given product could be attributed to consequent fragmentation of sildenafil due to elimination of piperazine group, subsequent elimination of sulfonic acid group, and subsequent elimination of ester-linked ethyl group. These characteristic peaks could reaffirm the presence of sildenafil in the product number 4 .

Fenbutrazate with a retention time of 12.72 was only screened in the structure of product number 8 .

Manufacturers of "Lipo 6" product, claimed to use caffeine and some synthetic mixtures to produce their slimming product, though the present study the presence of phendimetrazine in their mixture was screened, based on UV results and the presence of a peak at retention time of $2.20 \mathrm{~min}$. The existence of phendimetrazine was confirmed by examining the mass spectra of the product (Fig. 3). Phendimetrazine is 
Table 1: Brand name, the dosage form, retention times of the observed peaks and type of adulterants in studied products

\begin{tabular}{|c|c|c|c|c|}
\hline $\begin{array}{l}\text { Sample } \\
\text { number }\end{array}$ & Products brand name & Dosage form & $\begin{array}{l}\text { Retention } \\
\text { time (min) }\end{array}$ & Adulterants \\
\hline \multirow[t]{2}{*}{1} & Magic Slim & Dark brown powder in Hard cap & 8.93 & Phenolphthalein \\
\hline & & & 2.65 & Caffeine \\
\hline \multirow[t]{2}{*}{2} & Relacore & Light brown powder in Hard cap & 8.94 & Phenolphthalein \\
\hline & & & 3.59 & Sibutramine \\
\hline 3 & Natural Slimming & Dark brown powder (more) in Hard cap & 8.94 & Phenolphthalein \\
\hline \multirow[t]{2}{*}{4} & Fast Slimming & Very light brown powder in Hard cap & 2.19 & Sildenafil \\
\hline & & & 10.75 & Protriptyline \\
\hline \multirow[t]{2}{*}{5} & PX-1 & Dark brown powder in the Hard cap & 8.93 & Phenolphthalein \\
\hline & & & 2.65 & Caffeine \\
\hline \multirow[t]{3}{*}{6} & Slimming & Dark brown powder in Hard cap & 8.93 & Phenolphthalein \\
\hline & & & 4.5 & Sibutramine \\
\hline & & & 10.75 & Protriptyline \\
\hline \multirow[t]{2}{*}{7} & Natural max slimming & Dark brown powder in hard cap & 8.93 & Phenolphthalein \\
\hline & & & 4.5 & Sibutramine \\
\hline \multirow{3}{*}{8} & & & 4.5 & Sibutramine \\
\hline & & & 10.75 & Protriptyline \\
\hline & & & 12.77 & Fenbutrazate \\
\hline \multirow[t]{2}{*}{9} & Lipo-6 & The white oily liquid in Soft cap & 2.65 & Caffeine \\
\hline & & & 2.2 & Phendimetrazine \\
\hline \multirow[t]{3}{*}{10} & Weight Killer & $\begin{array}{l}\text { Relatively dark brown powder in Hard } \\
\text { cap }\end{array}$ & 2.65 & Caffeine \\
\hline & & & 5.3 & Docusate sodium \\
\hline & & & 9.3 & Docusate potassium \\
\hline 11 & Botanical Slimming & Oily liquid matte white in Soft cap & 8.93 & Phenolphthalein \\
\hline \multirow[t]{2}{*}{12} & Sample 12- not wrapped using specific form & Solid dark powder in Soft cap & 8.93 & Phenolphthalein \\
\hline & & & 2.65 & Caffeine \\
\hline \multirow[t]{2}{*}{13} & Feel Slim & $\begin{array}{l}\text { Relatively dark brown powder in Hard } \\
\text { cap }\end{array}$ & 8.93 & Phenolphthalein \\
\hline & & & 4.5 & Sibutramine \\
\hline 14 & Hodia-P57 & Relatively opaque oily liquid in Soft cap & 8.93 & Phenolphthalein \\
\hline \multirow[t]{2}{*}{15} & HydroxyStim & Light brown powder in Hard cap & 2.65 & Caffeine \\
\hline & & & 2.2 & Phendimetrazine \\
\hline
\end{tabular}

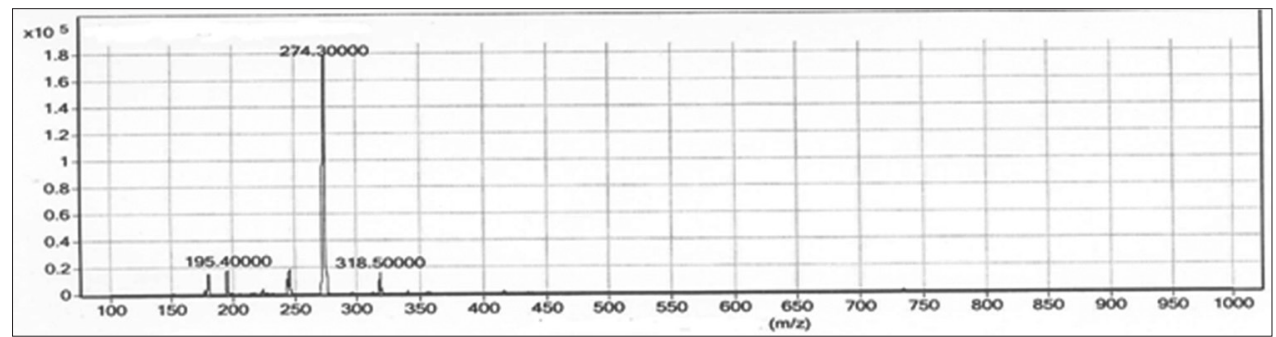

Fig. 1: Mass spectrum of phenolphthalein

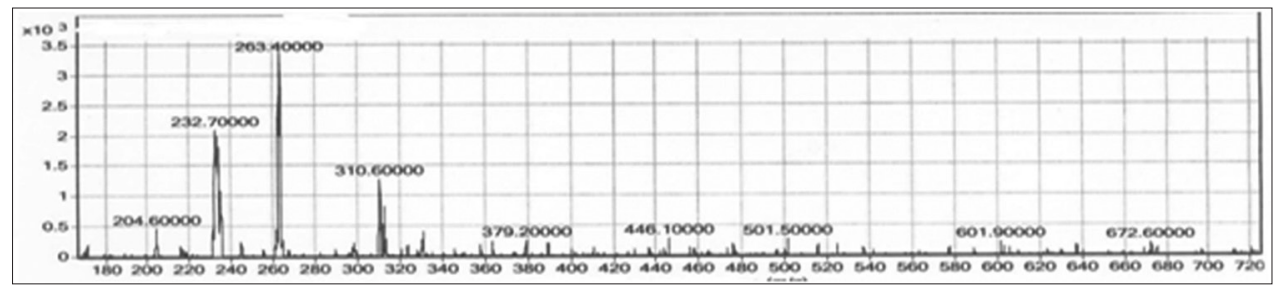

Fig. 2: Mass spectrum of protriptyline

an anti-appetite substance with the molecular mass of $341.3 \mathrm{Da}$. The molecular ion peak of phendimetrazine was identified at $\mathrm{m} / \mathrm{z} 340.6$ (obtained from mass analysis of the product), which could affirm addition of phendimetrazine in this product. This adulterant was also detected in the product number 15 .
The mass studies suggested the existence of sodium and potassium salts of docusate in product number 10 (Fig. 4). Sodium and potassium salts of docusate are using as laxatives in this slimming product. The molecular mass of docusate sodium and potassium is 444.6 and 460.7 $\mathrm{Da}$, respectively. Two peaks at $\mathrm{m} / \mathrm{z} 442$ and $\mathrm{m} / \mathrm{z} 290$ were found in the 


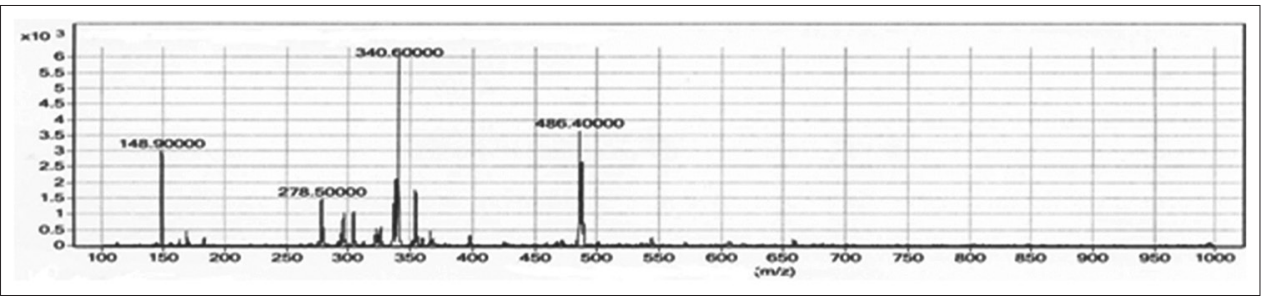

Fig. 3: Mass spectrum of phendimetrazine

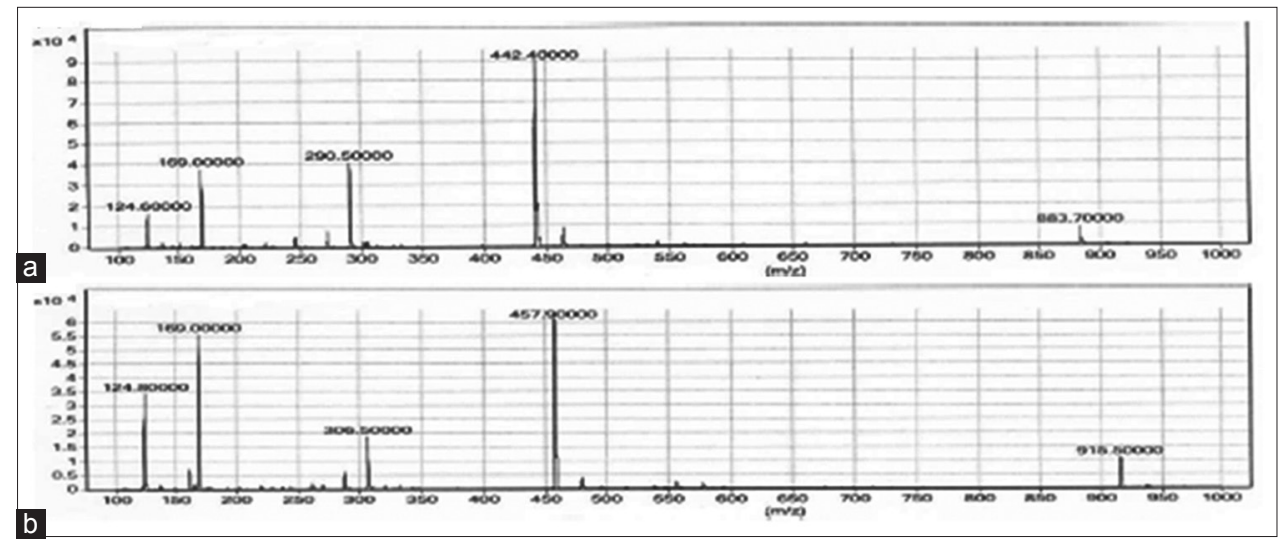

Fig. 4: (a) Mass spectrum of docusate sodium and (b) mass spectrum of docusate potassium

mass spectrum of product 10 . They could be attributed to the molecular ion peak of docusate sodium and its fragment due to loss of $\mathrm{CH}_{2} \mathrm{COOH}$ $\left(\mathrm{C}_{2} \mathrm{H}_{5}\right) \mathrm{CH}\left(\mathrm{CH}_{2}\right)_{3} \mathrm{CH}_{3}$ chain. Furthermore, two peaks at $\mathrm{m} / \mathrm{z} 458$ and $\mathrm{m} / \mathrm{z} 306$ were found in the mass spectrum of this product, respectively, corresponding to the molecular ion peak and fragmentation of docusate potassium due to elimination of $\mathrm{CH}_{2} \mathrm{COOH}\left(\mathrm{C}_{2} \mathrm{H}_{5}\right) \mathrm{CH}\left(\mathrm{CH}_{2}\right)_{3}$ $\mathrm{CH}_{3}$ chain. The same method of fragmentation of these two compounds (elimination of a same chain) could be due to the same origin of bath salts.

A comparison between the fragmentation of this sample and spectra of standard salts of docusate indicates that the presence of docusate might be more probable in this product (Fig. 4).

For further investigating, the presence of some other synthetic drugs in the products was examined in this study. The retention time of these synthetic compounds was found 5.54, 3.18, 2.07, and $1.58 \mathrm{~min}$ for levothyroxine, PTU, methamphetamine, and orlistat, while these peaks were not observed for the studied products. We could not found these adulterants in any of these products.

\section{CONCLUSION}

This study aimed to analyze the mostly used slimming products of Iran market and find out the presence of adulterants which are not mentioned on their labels. The major objective was to prepare both screening and confirmation procedures simultaneously in a simple and reliable method. The characteristics retention times in chromatographs in association with sufficient diagnostic ions in mass spectra of the samples helps to achieve this goal. Considering the number of slimming products available in global and Iran markets, few studies in Iran and several studies around the world proved the existence of synthetic mixtures in many slimming products $[20,26,27]$

In our study, Sibutramine, phenolphthalein, caffeine, fenbutrazate, phendimetrazine, sildenafil, and docusate $\mathrm{Na}$ and docusate $\mathrm{K}$ were identified as the synthetic adulterants in the slimming products. Substances such as those identified in this study, that are not declared on the label of weight loss food supplements can have very serious consequences on the quality of life of consumers and consumers are unaware of their side effects. Phenolphthalein was the most abundant adulterant which has been used in 11 cases of the 15 slimming products. Phenolphthalein is considered as a purgative chemical substance and can result in breast and ovary cancers among women [28,29]. Furthermore, 5 products included caffeine as a metabolism stimulator to increase the metabolism rate in the body and, as the result, losing weight $[30,31]$. Two products showed to include phendimetrazine as an anti-appetite substance, and fenbutrazate were used in one product as an anti-appetite substance, to Protriptyline, a tricyclic anti-depression drug, was found in 2 products.

In this study, docusate sodium and potassium, as purgatives [32], were found in one of the examined products. The LC-UV and LC-MS ranges proved that sildenafil was used in the "fast slimming" product; while descriptions on the box only mentioned addition of herbs to increase sexual sensations. It was the first time that the presence of docusate sodium and potassium and fenbutrazate is reported as adulterants in slimming products, which has been reported in this study. This study was successful in screening and confirming the presence of different common adulterants simultaneously in the slimming products. In general, due to the public interest in the extensive uses of weight loss medications, it is necessary to create awareness in these cases through public authorities.

\section{ACKNOWLEDGMENT}

Financial supports (Research Grant No. 92329) of Vice Chancellery for Research and Technology of Kermanshah University of Medical Sciences is acknowledged. This article resulted from the Pharm. D thesis of Sajad Fakhri, Major of Pharmacy, Kermanshah University of Medical Sciences, Kermanshah, Iran.

\section{REFERENCES}

1. Bonfrate L, Wang DQ, Garruti G, Portincasa P. Obesity and the risk and prognosis of gallstone disease and pancreatitis. Best Pract Res Clin Gastroenterol 2014;28:623-35.

2. Nerkar D, Mukherjee A, Metha BK, Banerjee S. Metabolic syndrome associated complications. Int J Pharm Pharm Sci 2015;7:22-5.

3. Basen-Engquist K, Chang M. Obesity and cancer risk: Recent review and evidence. Curr Oncol Rep 2011;13:71-6. 
4. Hamer M, Stamatakis E. Metabolically healthy obesity and risk of allcause and cardiovascular disease mortality. J Clin Endocrinol Metab 2012;97:2482-8

5. Matsuda M, Shimomura I. Roles of adiponectin and oxidative stress in obesity-associated metabolic and cardiovascular diseases. Rev Endocr Metab Disord 2014;15:1-10.

6. Sarnali TT, Md Moyenuddin PK. Obesity and disease association: A review. Anwer Khan Mod Med Coll J 2010;1:21-4.

7. Head GA, Lim K, Barzel B, Burke SL, Davern PJ. Central nervous system dysfunction in obesity-induced hypertension. Curr Hypertens Rep 2014;16:466.

8. Organization WH. Obesity and Overweight; 2013. Available from: http://www.Who.Int/mediacentre/factsheets/fs311/en. [Last accessed on 2013 Oct 11].

9. de Carvalho LM, Martini M, Moreira AP, de Lima AP, Correia D, Falcão T, et al. Presence of synthetic pharmaceuticals as adulterants in slimming phytotherapeutic formulations and their analytical determination. Forensic Sci Int 2011;204:6-12.

10. Yu Z, Wei Q, Fan Q, Wan C. A simple and convenient method for simultaneous determination of four major species of illegal additives in slimming health food. J Liq Chromatogr Relat Technol 2010;33:452-61.

11. Kim HJ, Lee JH, Park HJ, Cho SH, Cho S, Kim WS, et al. Monitoring of 29 weight loss compounds in foods and dietary supplements by LC-MS/MS. Food Addit Contam Part A Chem Anal Control Expo Risk Assess 2014:31:777-83

12. Lv D, Cao Y, Lou Z, Li S, Chen X, Chai Y, et al. Rapid on-site detection of ephedrine and its analogues used as adulterants in slimming dietary supplements by TLC-SERS. Anal Bioanal Chem 2015;407:1313-25.

13. Miao L, Liu Y, Li H, Qi Y, Lu F. Two-dimensional correlation infrared spectroscopy applied to the identification of ephedrine and pseudoephedrine in illegally adulterated slimming herbal products. Drug Test Anal 2017;9:221-9.

14. Ozdemir B, Sahin I, Kapucu H, Celbis O, Karakoc Y, Erdogan S, et al. How safe is the use of herbal weight-loss products sold over the internet? Hum Exp Toxicol 2013;32:101-6.

15. Tukker A, Visscher TL, Picavet HS. Overweight and health problems of the lower extremities: Osteoarthritis, pain and disability. Public Health Nutr 2009;12:359-68.

16. Yuen YP, Lai CK, Poon WT, Ng SW, Chan AY, Mak TW, et al. Adulteration of over-the-counter slimming products with pharmaceutical analogue-an emerging threat. Hong Kong Med J 2007;13:216-20.

17. Posadzki P, Watson L, Ernst E. Contamination and adulteration of herbal medicinal products (HMPs): An overview of systematic reviews. Eur J Clin Pharmacol 2013;69:295-307.
18. Ioset JR, Raoelison GE, Hostettmann K. Detection of aristolochic acid in Chinese phytomedicines and dietary supplements used as slimming regimens. Food Chem Toxicol 2003;41:29-36.

19. Morris CA, Avorn J. Internet marketing of herbal products. JAMA 2003;290:1505-9.

20. Khazan M, Hedayati M, Kobarfard F, Askari S, Azizi F. Identification and determination of synthetic pharmaceuticals as adulterants in eight common herbal weight loss supplements. Iran Red Crescent Med J 2014; $16: \mathrm{e} 15344$

21. Lobstein T, Jackson-Leach R. Child overweight and obesity in the USA: Prevalence rates according to IOTF definitions. Int J Pediatr Obes 2007;2:62-4.

22. Majnooni MB, Jalili R, Mohammadi B, Miraghaee SS, Fakhri S, Bahrami G. Development and validation of a new method for determination of topiramate in bulk and pharmaceutical formulation using high performance liquid chromatography-UV detection after precolumn derivatization. J Rep Pharm Sci 2014;3:179-83.

23. Mohammadi B, Tammari E, Fakhri S, Bahrami G. Applicability of LC-MS/MS to optimize derivatization of topiramate with FMOC-cl using reacted/intact drug ratio. J Chromatogr B Analyt Technol Biomed Life Sci 2013;928:32-6.

24. Abe AM, Hein DJ, Gregory PJ. Regulatory alerts for dietary supplements in canada and the united states, 2005-13. Am J Health Syst Pharm 2015;72:966-71.

25. Bollapragada MK, Shantaram M, Kumar S. Obesity: Development, epidemiology, factors affecting, quantity, health hazards, management, and natural treatment-A review. Int J Pharm Pharm Sci 2017;9:12-26.

26. Venhuis B, Zwaagstra M, Van den Berg J, Wagenaar H, Van Riel A, Barends D, et al. Trends in Drug Substances Detected in Illegal Weight-loss Medicines and Dietary Supplements, RIVM Report. No. 370030002; 2009. p. 1-37.

27. Word Health Organization. WHO Guidelines on Safety Monitoring of Herbal Medicines in Pharmacovigilance Systems. Geneva: WHO; 2004

28. Markel G. Method of Diagnosing Cancer. Google Patents; 2010

29. Roerig JL, Steffen KJ, Mitchell JE, Zunker C. Laxative abuse: Epidemiology, diagnosis and management. Drugs 2010;70:1487-503.

30. Westerterp-Plantenga MS. Green tea catechins, caffeine and bodyweight regulation. Physiol Behav 2010;100:42-6.

31. Yang CS, Zhang J, Zhang L, Huang J, Wang Y. Mechanisms of body weight reduction and metabolic syndrome alleviation by tea. Mol Nutr Food Res 2016;60:160-74.

32. Verghese TS, Futaba K, Latthe P. Constipation in pregnancy. Obstet Gynaecol 2015;17:111-5. 